\title{
Magnetic clouds' structure in the magnetosheath as observed by Cluster and Geotail: four case studies
}

\author{
L. Turc ${ }^{1}$, D. Fontaine ${ }^{1}$, P. Savoini ${ }^{1}$, and E. K. J. Kilpua ${ }^{2}$ \\ ${ }^{1}$ Ecole Polytechnique, CNRS, Sorbonne Universités, UPMC Univ Paris 06, Univ Paris-Sud, UMR7648, \\ Laboratoire de Physique des Plasmas, 91128 Palaiseau, France \\ ${ }^{2}$ Department of Physics, University of Helsinki, P.O. Box 64, 00014 Helsinki, Finland \\ Correspondence to: L. Turc (lucile.turc@1pp.polytechnique.fr)
}

Received: 6 May 2014 - Revised: 29 July 2014 - Accepted: 5 September 2014 - Published: 15 October 2014

\begin{abstract}
Magnetic clouds (MCs) are large-scale magnetic flux ropes ejected from the Sun into the interplanetary space. They play a central role in solar-terrestrial relations as they can efficiently drive magnetic activity in the near-Earth environment. Their impact on the Earth's magnetosphere is often attributed to the presence of southward magnetic fields inside the MC, as observed in the upstream solar wind. However, when they arrive in the vicinity of the Earth, MCs first encounter the bow shock, which is expected to modify their properties, including their magnetic field strength and direction. If these changes are significant, they can in turn affect the interaction of the $\mathrm{MC}$ with the magnetosphere. In this paper, we use data from the Cluster and Geotail spacecraft inside the magnetosheath and from the Advanced Composition Explorer (ACE) upstream of the Earth's environment to investigate the impact of the bow shock's crossing on the magnetic structure of MCs. Through four example MCs, we show that the evolution of the MC's structure from the solar wind to the magnetosheath differs largely from one event to another. The smooth rotation of the MC can either be preserved inside the magnetosheath, be modified, i.e. the magnetic field still rotates slowly but at different angles, or even disappear. The alteration of the magnetic field orientation across the bow shock can vary with time during the MC's passage and with the location inside the magnetosheath. We examine the conditions encountered at the bow shock from direct observations, when Cluster or Geotail cross it, or indirectly by applying a magnetosheath model. We obtain a good agreement between the observed and modelled magnetic field direction and shock configuration, which varies from quasiperpendicular to quasi-parallel in our study. We find that the variations in the angle between the magnetic fields in the
\end{abstract}

solar wind and in the magnetosheath are anti-correlated with the variations in the shock obliquity. When the shock is in a quasi-parallel regime, the magnetic field direction varies significantly from the solar wind to the magnetosheath. In such cases, the magnetic field reaching the magnetopause cannot be approximated by the upstream magnetic field. Therefore, it is important to take into account the conditions at the bow shock when estimating the impact of an MC with the Earth's environment because these conditions are crucial in determining the magnetosheath magnetic field, which then interacts with the magnetosphere.

Keywords. Interplanetary physics (planetary bow shocks) - magnetospheric physics (magnetosheath; solar-windmagnetosphere interactions)

\section{Introduction}

Coronal mass ejections (CMEs) are tremendous eruptions in the solar corona during which the solar magnetic field and plasma are ejected into the interplanetary medium. Interplanetary coronal mass ejections (ICMEs), their counterparts in the heliosphere, are central in driving disturbances in the near-Earth environment (Richardson et al., 2001, 2002; Echer et al., 2008; Yermolaev et al., 2012). According to the observations near the first Lagrangian point of the SunEarth system (L1), around $30 \%$ of the ICMEs display a flux-rope-like magnetic field configuration (Richardson and Cane, 2010) and are referred to as magnetic clouds (MCs). This subset of ICMEs is primarily defined by an enhanced magnetic field, relative to the surrounding solar wind, which 
smoothly rotates over a large angle, and a lower temperature (Burlaga et al., 1981).

MCs are particularly geoeffective structures, i.e. they efficiently trigger geomagnetic storms (see, for example, Zhang et al., 2004; Huttunen et al., 2005). Their geoeffectivity can generally be related to the presence of a long-lasting southward magnetic field. This stems from the fact that the Earth's magnetic field is essentially northward in the equatorial plane, and thus a southward magnetic field is favourable to reconnection at the magnetopause. However, the correlation between southward fields inside the MC and the occurrence of a magnetic storm is not one to one. For example, statistical studies show that no storm is associated with a substantial fraction of MCs with southward fields, from $18 \%$ (Zhang et al., 2004) to $28 \%$ (Huttunen et al., 2005; Gopalswamy et al., 2008) of the events. Therefore, a better understanding of the interaction of MCs with the Earth's environment is needed to refine the prediction of their consequences inside the magnetosphere, and thus improve space weather forecasting.

When the relationship between the MC's parameters and its geoeffectivity is not straightforward, various scenarios can be considered. Geomagnetic storms occurring during MCs with only northward fields are frequently attributed to the sheath preceding the MC (Huttunen et al., 2005). The magnetic fields at the leading or the trailing edge of the $\mathrm{MC}$ are also sometimes invoked to account for the effects observed inside the magnetosphere (Zhang et al., 2004). Some recent studies hint at the pivotal role played by the magnetosheath in the solar-wind-magnetosphere coupling, which is still poorly understood (Šafránková et al., 2009; Lavraud et al., 2013; Turc et al., 2014).

The magnetosheath is the region bounded by the bow shock and the magnetopause in which the shocked solar wind is deflected around the magnetosphere. The global physics of this region have been well understood since the early work of Spreiter et al. (1966), and have been extensively confirmed by spacecraft observations. It is well known that the bow shock crossing alters the solar wind properties. Its speed decreases, while the density, temperature and magnetic field strength increase. Downstream of the bow shock, the magnetic field lines pile up and drape around the magnetopause. More recent studies suggest that other phenomena, such as field-flow coupling, may also play a role in modifying the magnetic field orientation inside the magnetosheath (Longmore et al., 2006). It is eventually this modified magnetic field, and not the interplanetary magnetic field (IMF) upstream of the bow shock, which interacts and possibly reconnects with the Earth's magnetic field.

Šafránková et al. (2009) focus on the north-south component of the IMF, $B_{z}$, which is crucial for solar-windmagnetosphere coupling, and study the probability of finding the same sign of $B_{z}$ upstream and downstream of the bow shock. They find that this probability is very low: the sign of $B_{z}$ is preserved only $12 \%$ of the time. During low Alfvén
Mach number conditions, which are often associated with MCs, accelerated flows, sometimes exceeding the solar wind speed, can be observed on the flanks of the magnetosheath and are attributed to the draping of the field lines (Lavraud and Borovsky, 2008; Lavraud et al., 2013). Such modifications of the magnetic field and velocity downstream of the bow shock are expected to affect the coupling between the solar wind and the magnetosphere (Lavraud and Borovsky, 2008).

However, to our knowledge, these effects are not taken into account when studying the geoeffectivity of MCs. Usually, only their properties upstream of the bow shock are considered (Zhang et al., 2004; Huttunen et al., 2005; Gopalswamy et al., 2008). Yet the physics taking place at the bow shock and inside the magnetosheath should apply to MCs as well as the quiet solar wind. The bow shock's crossing and the propagation downstream towards the magnetopause should alter the MCs' smoothly rotating magnetic field, and thus its impact on the magnetosphere.

A model of the magnetosheath magnetic field designed for MC studies has recently been introduced by Turc et al. (2014). In this paper, they investigate the impact of the bow shock's crossing on synthetic flux ropes. They find for certain flux ropes' orientations that the direction of the MC's magnetic field can vary significantly from the solar wind to the magnetosheath, sometimes exceeding $60^{\circ}$ and occasionally causing a reversal of the $B_{z}$ component. This is obtained in the dayside magnetosheath independently of the field line draping closer to the magnetopause. These variations are related to the configuration, quasi-parallel or quasiperpendicular, encountered at the bow shock. In addition, Turc et al. (2014) determine the location of anti-parallel fields, that is, favourable to reconnection, along the magnetopause and find patterns very different from that expected from the MC's magnetic field orientation upstream of the shock. For example, an initially northward field during an MC can turn south in a part of the magnetosheath and result in a region of anti-parallel fields near the subsolar point. Therefore, Turc et al. (2014) suggest that the impact of an MC on the magnetosphere can be strongly modified by the processes occurring at the shock and inside the magnetosheath.

Although MCs have been the topic of intensive scrutiny in the solar wind, little is known of their properties in the Earth's magnetosheath. One of the reasons for this is that spacecraft observations in this region are sporadic. Satellites dedicated to the study of the Earth's magnetosphere, such as Cluster (Escoubet et al., 1997), the Geomagnetic Tail Lab (GEOTAIL) (Nishida, 1994), Double Star (Liu et al., 2005) or the Time History of Events and Macroscale Interactions during Substorms (THEMIS) (Angelopoulos, 2008), occasionally cross this region, but their stay in the magnetosheath generally lasts only for a few hours. As this has to coincide with the arrival of an MC in the vicinity of Earth, which has 

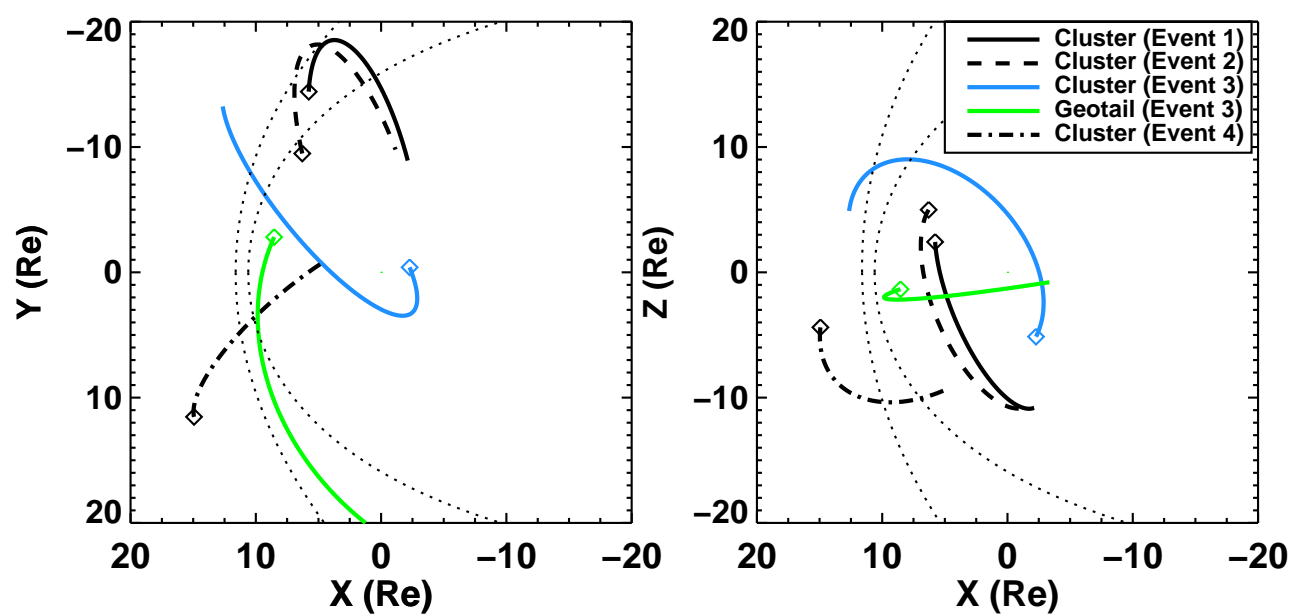

Figure 1. Orbits of the spacecraft during the four studied events in the GSE $x-y$ (left panel) and $x-z$ (right panel) planes. The diamonds indicate the location of the spacecraft at the beginning of the event. The black dotted lines indicate the position of the Jeřáb et al. (2005) bow shock model and of the Shue et al. (1998) magnetopause model for typical solar wind conditions $\left(n=6 \mathrm{~cm}^{-3}, V=400 \mathrm{~km} \mathrm{~s}-1, B=5 \mathrm{nT}\right.$, $\left.B_{z}=0 \mathrm{nT}\right)$.

an even smaller occurrence, MCs are seldom observed in the magnetosheath.

This paper focuses on correlations between observations in the solar wind upstream of the bow shock and in the magnetosheath during $\mathrm{MC}$ events. The aim is to investigate whether the magnetic structure of MCs is modified across the bow shock and in the magnetosheath, and to quantitatively compare the spacecraft observations to the predictions of the magnetosheath model developed by Turc et al. (2014). We have selected four typical MC events during which one or several spacecraft are located inside the Earth's magnetosheath, at least for several hours during the MC's passage. We focus here on the magnetic structure of these MCs, i.e. the smooth rotation of their magnetic field, and how it evolves from the solar wind to the magnetosheath. Each of the four cases corresponds to a different evolution of the magnetic structure across the bow shock and inside the magnetosheath, and illustrates a typical example of magnetosheath observations during MCs.

The paper is organised as follows: in Sect. 2 we briefly introduce the different data sets used in this work. The four example MCs are described in Sect. 3. In Sect. 4, we investigate the conditions encountered at the bow shock, and compare our results to the Turc et al. (2014) magnetosheath model in Sect. 5. Section 6 concludes this paper with a summary and a discussion.

\section{Data sets}

To investigate how the structure of MCs evolves from the solar wind to the magnetosheath, we need simultaneous observations in these two regions. The solar wind upstream of the Earth is continuously monitored by the Advanced
Composition Explorer (ACE) (Stone et al., 1998) spacecraft, located near L1. Other data sets, such as the measurements from the Wind spacecraft (Acuña et al., 1995) or the OMNI data set propagated to Earth's bow shock nose, can also be used as solar wind monitors. However, during some of the events presented in the next sections, the Wind spacecraft was not located upstream of Earth's environment, and in one of the studied intervals the OMNI propagated data set contains a large data gap.

Therefore, we use ACE Magnetic Field Experiment (MAG) (Smith et al., 1998) data as a monitor of the magnetic structure of MCs in the unperturbed solar wind. We assume that all the large-scale structures observed in L1 actually reach the Earth's environment. As ACE is located close to the Sun-Earth line, this is generally true. The data are obtained through the CDAWeb service (http://cdaweb.gsfc.nasa.gov/ spphys/). They are shifted in time in order to account for the propagation from L1 to the magnetosheath. The delay is obtained by dividing the distance between ACE and the spacecraft inside the magnetosheath in the $x$ direction by the solar wind speed along $x$. Using a constant time lag throughout the studied events is sufficient for the purpose of our study, as we focus on long-lasting and large-scale structures. In the following, we will only display time-shifted ACE data, and the reference time will be given by Cluster's observations.

In the magnetosheath, we use data from the Cluster and the Geotail spacecraft when available in this region. However, since the spacecraft generally do not remain in the magnetosheath during the entire MC events, observations in the solar wind and/or the magnetosphere are also displayed. The magnetic field measurements are obtained from the FluxGate Magnetometer (FGM) (Balogh et al., 1997) aboard Cluster and the Magnetic Field (MGF) instrument aboard Geotail (Kokubun et al., 1994). All data from ACE, Cluster and 
Geotail shown in the following sections are 1 min averages, unless otherwise indicated. The magnetic field components are given in the geocentric solar ecliptic (GSE) frame.

\section{Description of the events}

In this section, we present four MCs observed simultaneously in the solar wind and the magnetosheath. They have been selected as the most representative ones from a database of about $30 \mathrm{MC}$ events during which spacecraft observations are available inside the magnetosheath. These four events comprise rather long intervals of magnetosheath measurements, and generally also bow shock crossings. Each of them illustrates a different behaviour of the MC's structure inside the magnetosheath.

The orbits of Cluster and Geotail during the studied intervals are displayed in Fig. 1, in the GSE $x-y$ (left panel) and the $x-z$ (right panel) planes. The diamonds indicate the position of the spacecraft at the beginning of the events. As a reference, the dotted lines indicate the Jeřáb et al. (2005) bow shock and the Shue et al. (1998) magnetopause models calculated for average solar wind conditions, namely $n=6 \mathrm{~cm}^{-3}$, $V=400 \mathrm{~km} \mathrm{~s}^{-1}, B=5 \mathrm{nT}$ and $B_{z}=0 \mathrm{nT}$. Figure 1 shows that the spacecraft provide us with a rather good coverage of the dayside magnetosheath during these four MCs.

\subsection{Event 1: MC's structure unchanged in the magnetosheath}

Figure 2 shows the observations of ACE (time shifted to the bow shock) in the solar wind (black curves) during $32 \mathrm{~h}$ starting from 20 May 2005 at 00:00 UT. Just after 04:00 UT on 20 May 2005, as indicated by the vertical dotted line, the magnetic field strength increases in the solar wind (top panel), corresponding to the beginning of the sheath of an MC. This enhancement of the magnetic field strength is accompanied by a weak shock observed on the solar wind speed (not shown). The MC arrives around 07:15 UT on 20 May 2005 and its front and rear edges are indicated by the vertical dotted-dashed lines. Between these two boundaries, we observe a slow variation of the magnetic field magnitude and components (see Fig. 2a, b, c and d), or equivalently a smooth rotation of the $\theta$ and $\varphi$ angles (Fig. 2e and f). These angles are defined as follows: $\cos \theta=B_{z} / B$ and $\cos \varphi=B_{x} /\left(\sqrt{B_{x}^{2}+B_{y}^{2}}\right)$.

During this event, Cluster (blue curves) is located close to the Earth's bow shock (solid black curves in Fig. 1). During the few hours preceding the shock and during the sheath of the MC, the spacecraft remains mostly in the solar wind, as evidenced by the magnetic field magnitude (blue curve, Fig. 2a), which is similar to that observed by ACE. After the beginning of the MC, that is, after 07:15 UT, and until 17:00 UT on 20 May 2005, Cluster crosses the bow shock several times and then moves from the solar wind to the magnetosheath, as indicated by the sharp increases in the magnetic field strength due to compression at the bow shock. After 17:00 UT, the spacecraft remains in the magnetosheath and reaches the magnetopause around 04:00 UT on 21 May 2005. The transition from the magnetosheath to the magnetosphere can be seen on the plasma measurements (not shown).

We now focus on the times when Cluster is located in the magnetosheath. Like the magnetic field strength, the magnetic field components (Fig. 2b, c and d) increase in absolute value from the solar wind to the magnetosheath because of the compression. However, Fig. 2e and f show that even though Cluster is located downstream of the bow shock, it observes roughly the same magnetic field direction as ACE. In particular, the bow shock crossings which are clearly seen on the magnetic field strength cannot be identified on $\theta$ and $\varphi$. Starting from 19:00 UT, we observe a discrepancy between the magnetic field directions measured by ACE and Cluster, which increases as Cluster moves closer to the magnetopause. This deviation is most likely due to the draping of the field lines around the magnetosphere, which progressively alters the magnetic field direction when approaching the magnetopause, and becomes more visible when moving farther tailward. Here Cluster is located at rather high latitudes, where the draping can be important. Another small variation (below $20^{\circ}$ ) is also observed between 09:00 and 11:00 UT on $\varphi$ (Fig. 2f), although Cluster remains close to the bow shock.

The bottom panel of Fig. 2 displays the angle $\psi$ between the magnetic field directions measured by ACE and by Cluster. This angle allows us to quantify the variation of the magnetic field direction from the solar wind to the magnetosheath with a single parameter. Contrary to the other quantities shown in Fig. 2, the angle $\psi$ is calculated from 5 min averages, because it depends on both ACE and Cluster measurements and thus on the time shift applied on the solar wind data set. We estimated that our propagation method leads to errors of $\pm 5 \mathrm{~min}$, due to the difference between the minimum and maximum speed during the event. Therefore, we compute the angle $\psi$ on 5 min averages of the magnetic field data in order to reduce the errors due to the constant time shift. The peak observed at 16:00 UT is due to a sharp variation of the magnetic field encompassed in the MC's smooth rotation.

We now focus on the global trend of $\psi$. The values of $\psi$ during the MC show that the variation of the magnetic field direction is mostly below $20^{\circ}$, that is, the magnetic structure of the MC observed in the magnetosheath is roughly the same as in the solar wind. We note, however, that $\psi$ sometimes increases; for example, between 09:00 and 11:00 UT, $\psi$ remains around $20^{\circ}$ for $2 \mathrm{~h}$. Also, after 00:00 UT on 21 May 2005, $\psi$ increases up to $40^{\circ}$ near the magnetopause, which is crossed around 04:00 UT on 21 May 2005. 


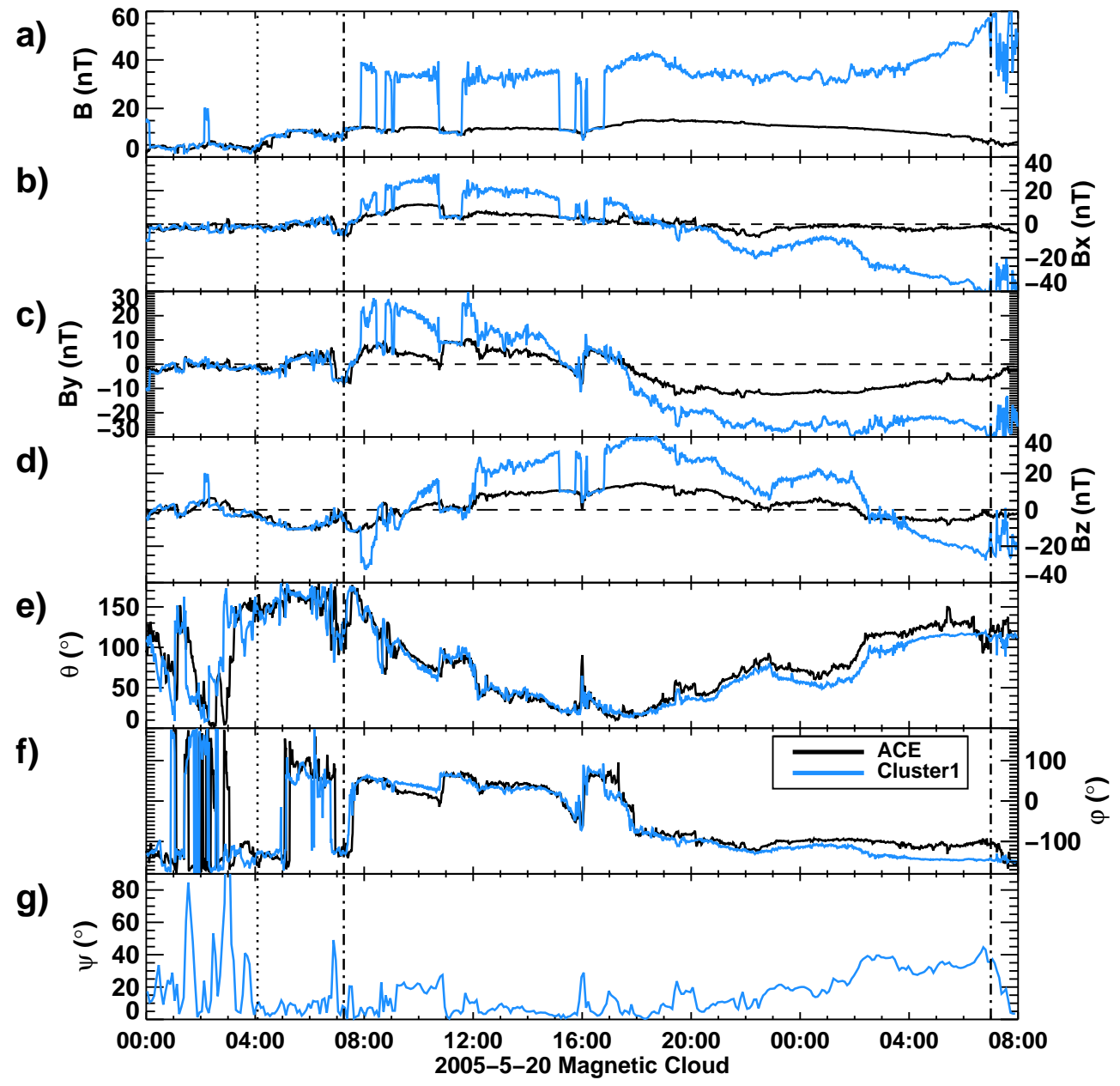

Figure 2. 20 May 2005 MC: observations from ACE (black) and Cluster (blue), in GSE coordinates. Vertical dotted line: beginning of the sheath of the MC. Vertical dotted-dashed lines: beginning and end of the MC. From top to bottom: magnetic field strength; $B_{x} ; B_{y} ; B_{z} ; \theta$; $\varphi$ (see text for definition of $\theta$ and $\varphi$ ); and $\psi$, the angle between the magnetic field observed by Cluster and by ACE.

\subsection{Event 2: temporal variation of the MC's structure}

The second MC that we will study here features one of the most extreme solar wind events of solar cycle 23 and has been the subject of detailed studies from the eruption of the CME on the Sun to its consequences on Earth's environment (see, for example, Dasso et al., 2009; Bisi et al., 2010). The sheath of this MC arrives around 02:40 UT on 15 May 2005 at Earth's bow shock (dotted line in Fig. 3). As can be seen in the top panel of Fig. 3, the magnetic field strength observed in the solar wind by ACE (black curve) increases up to $55 \mathrm{nT}$ at the beginning of the MC, indicated by the dotted-dashed line, that is, about 10 times higher than its usual value in the solar wind. Figure 3 shows the magnetic field observations from 01:00 UT on 15 May 2005 to 13:00 UT on 16 May 2005. Although it takes more than $48 \mathrm{~h}$ for this $\mathrm{MC}$ to propagate past $\mathrm{ACE}$, we only display here the part of the event where we have observations from Cluster in the solar wind or in the magnetosheath. Around 11:00 UT on
16 May 2005, Cluster crosses the magnetopause and moves into the magnetosphere.

As in the previous case, Cluster (blue curves) is in the solar wind when it measures the same magnetic field as ACE and in the magnetosheath when the magnetic field is compressed. Its orbit is drawn as black dashed curves in Fig. 1. After the beginning of the MC, Cluster crosses the bow shock numerous times, and finally stays in the magnetosheath after 22:30 UT. The investigation of the magnetic field direction, shown by the angles $\theta$ and $\varphi$ (Fig. 3b and c), reveals that the magnetosheath observations of this $\mathrm{MC}$ are divided into two distinct parts. First, from 06:00 to 17:00 UT on 15 May 2005, the magnetic field direction strongly differs from that in the solar wind each time Cluster is located downstream of the bow shock. The discrepancies can exceed $60^{\circ}$ and are largest on the angle $\varphi$. Depending on the considered magnetosheath interval, the direction of rotation of the angles $\theta$ and $\varphi$ varies. Moreover, the magnetic field direction and magnitude display large fluctuations inside the magnetosheath, in particular 
between 09:00 and 12:00 UT, while the upstream magnetic field still rotates slowly. Then, during the latter part of the event, that is, after 17:00 UT, we get back to the same situation as in Event 1, where the magnetic field direction is strikingly similar to that in the solar wind.

The bottom panel of Fig. 3 displays the angle $\psi$ between the magnetic field vectors at ACE and at Cluster. During the first part of the event, $\psi$ ranges between 30 and $70^{\circ}$ each time Cluster is in the magnetosheath, but afterwards it falls below $20^{\circ}$. One of the factors that might explain the variations in $\psi$ within the MC is the distance of Cluster from the bow shock. In the first interval, Cluster remains near the bow shock, as evidenced by the multiple shock crossings. Then, in the second interval, Cluster traverses across the magnetosheath to the magnetopause. However, the multiple bow shock crossings observed between 19:00 and 22:00 UT indicate that the spacecraft is still rather close to the bow shock during the first few hours of the second part of the event. Therefore, the distance to the bow shock alone cannot explain the differences between the first and the second parts of the event.

\subsection{Event 3: spatial variation of the MC's structure}

Figure 4 shows observations of three different spacecraft (ACE, Cluster and Geotail) of an MC, between 21 April 2001 at 15:00 UT and 22 April 2001 at 16:00 UT. ACE was located at L1, while Cluster and Geotail were found in the opposite flanks of the magnetosheath, as can be seen in Fig. 1. ACE data, in the solar wind, are drawn in black in Fig. 4. The beginning of the sheath of the MC is indicated by the dotted line, and the dotted-dashed line marks the front edge of the MC. As in Event 2, we do not show the entire MC, but only the part when data are available in the dayside magnetosheath or close by $\left(X_{\mathrm{GSE}} \gtrsim-3 R_{\mathrm{E}}\right)$, that is, until 16:00 UT on 22 April 2001. The usual magnetic field signatures of the MC are again clearly seen in ACE measurements: the higher magnetic field strength (Fig. 4a) and the smooth rotation of its direction (Fig. $4 \mathrm{~b}$ and c).

At the beginning of the event, from 15:00 to 22:00 UT on 21 April 2001, Cluster is located inside the magnetosphere, as shown by its orbit drawn in blue in Fig. 1. During the sheath of the cloud, around 22:00 UT on 21 April 2001, Cluster crosses the magnetopause and enters the dawnside magnetosheath, where it travels for several hours. The spacecraft observes the arrival of the MC around 00:30 UT on 22 April 2001 (dotted-dashed line in Fig. 4), then encounters the bow shock several times from 06:00 to 08:30 UT (see the blue curve in Fig. 4a). After 08:30 UT on 22 April 2001, Cluster remains almost continuously in the solar wind until the end of the MC and observes the same structure as ACE. Therefore, it provides us with only a few hours of magnetosheath observations during this event, from 00:30 to 08:30 UT. When comparing Cluster magnetosheath measurements to ACE solar wind observations, we note again that the MC's magnetic field direction observed in the magnetosheath is very similar to that at $\mathrm{L} 1$ (Fig. $4 \mathrm{~b}$ and c). The values of $\psi$ (blue curve in Fig. 4d) are below $20^{\circ}$ during the entire MC, showing that the variations in the magnetic field direction are very small, even downstream of the bow shock. Therefore, this event as observed by Cluster is rather similar to Event 1 and to the second part of Event 2.

At the MC's arrival, shortly before 01:00 UT on 22 April 2001 (dotted-dashed line), Geotail (green curves) makes an inbound crossing of the bow shock and remains in the magnetosheath during the entire event. While Cluster is located on the dawnside of the Northern Hemisphere, Geotail probes the duskside magnetosheath, close to the ecliptic plane (see the green curve in Fig. 1). During this event, Geotail travels from the dayside to the nightside magnetosheath, and crosses the terminator plane (i.e. $X_{\mathrm{GSE}}=0$ ) around 11:30 UT. Because the magnetosheath properties change when moving farther tailward, we concentrate on the observations from 00:30 to 16:00 UT on 22 April 2001, although the MC lasts until 01:00 UT the next day. Figure 4b shows that the angle $\theta$ observed by Geotail is similar to Cluster and ACE measurements, with the exception of the first $2 \mathrm{~h}$ of the MC, from 01:00 to 03:00 UT on 22 April 2001, when $\theta$ displays a variation of about $30^{\circ}$. For the angle $\varphi$ (Fig. 4c), however, the discrepancies between Geotail and the other data sets are much larger. From 01:00 to 16:00 UT on 22 April 2001, the green curve in Fig. 4c shows a similar rotation of the magnetic field along $\varphi$, but shifted by about $40^{\circ}$ relative to that observed by ACE and Cluster.

The angle $\psi$ (green curve in Fig. 4d) varies from $50^{\circ}$ at the beginning of the $\mathrm{MC}$ to $10^{\circ}$ at the end of the event. In particular, we note that, between 01:00 and 06:00 UT on 22 April 2001, when Cluster and Geotail are both located inside the magnetosheath, the values of $\psi$ differ largely from one observation point to another. In the dawnside, Cluster encounters roughly the same MC's structure as ACE, whereas, in the duskside, Geotail observes a magnetic field direction which is shifted by about $40^{\circ}$ but still rotates smoothly. Therefore, the magnetic field direction in the magnetosheath can differ significantly, depending on the location inside this region.

\subsection{Event 4: loss of the MC's structure}

Observations of the ACE spacecraft (black curves) during the 22 January $2004 \mathrm{MC}$ are displayed in Fig. 5. The sheath of the MC arrives shortly after 01:30 UT (dotted line), when the magnetic field strength increases from 5 to $25 \mathrm{nT}$ in the solar wind (Fig. 5a). The smooth rotation of the MC begins around 10:40 UT (dotted-dashed line), as can be seen in the magnetic field direction measured by ACE (Fig. 5b and c). Cluster (blue curves) is first located in the solar wind since it observes a magnetic field magnitude very similar to that at ACE during the sheath and the beginning of the MC (see also the dotted-dashed curves in Fig. 1). It then moves to the magnetosheath. However, the time of the bow shock 


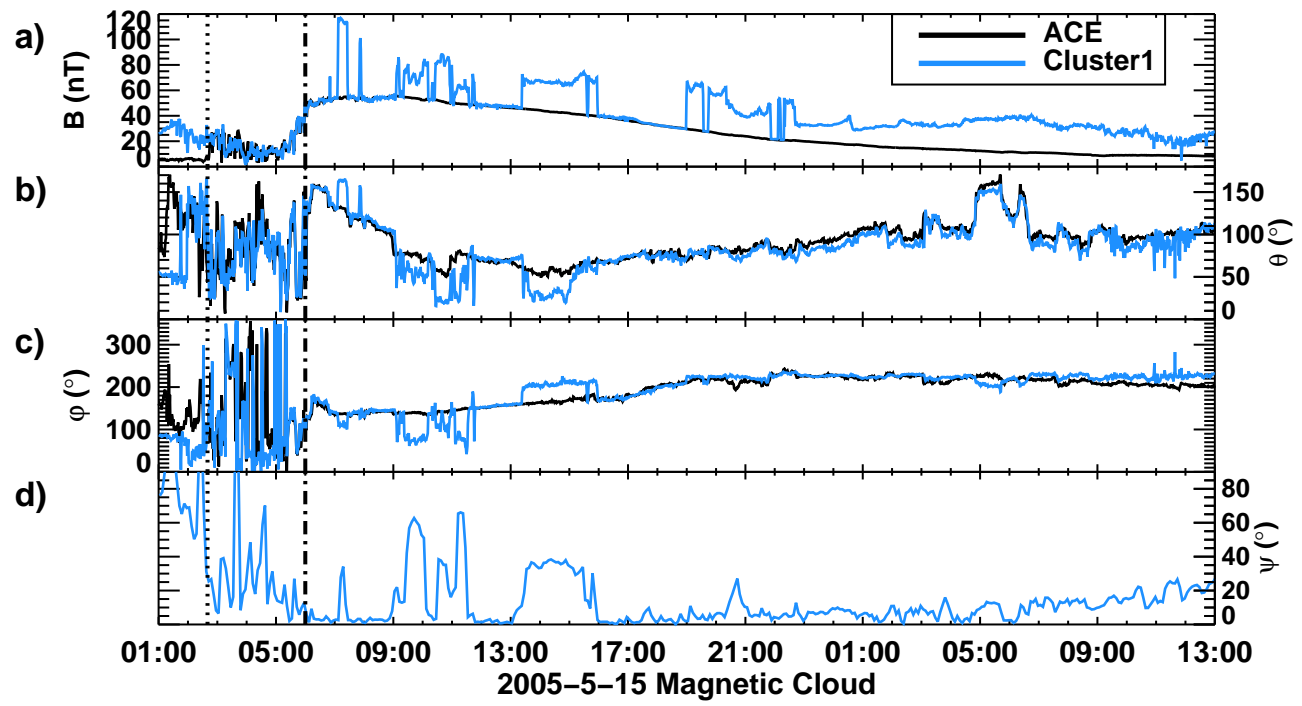

Figure 3. 15 May 2005 MC: observations from ACE (black) and Cluster (blue), in GSE coordinates. Vertical dashed line: beginning of the sheath of the MC. Vertical dotted dashed line: beginning of the MC. From top to bottom: magnetic field strength; $\theta ; \varphi$; and $\psi$, the angle between the magnetic field observed by Cluster and by ACE.

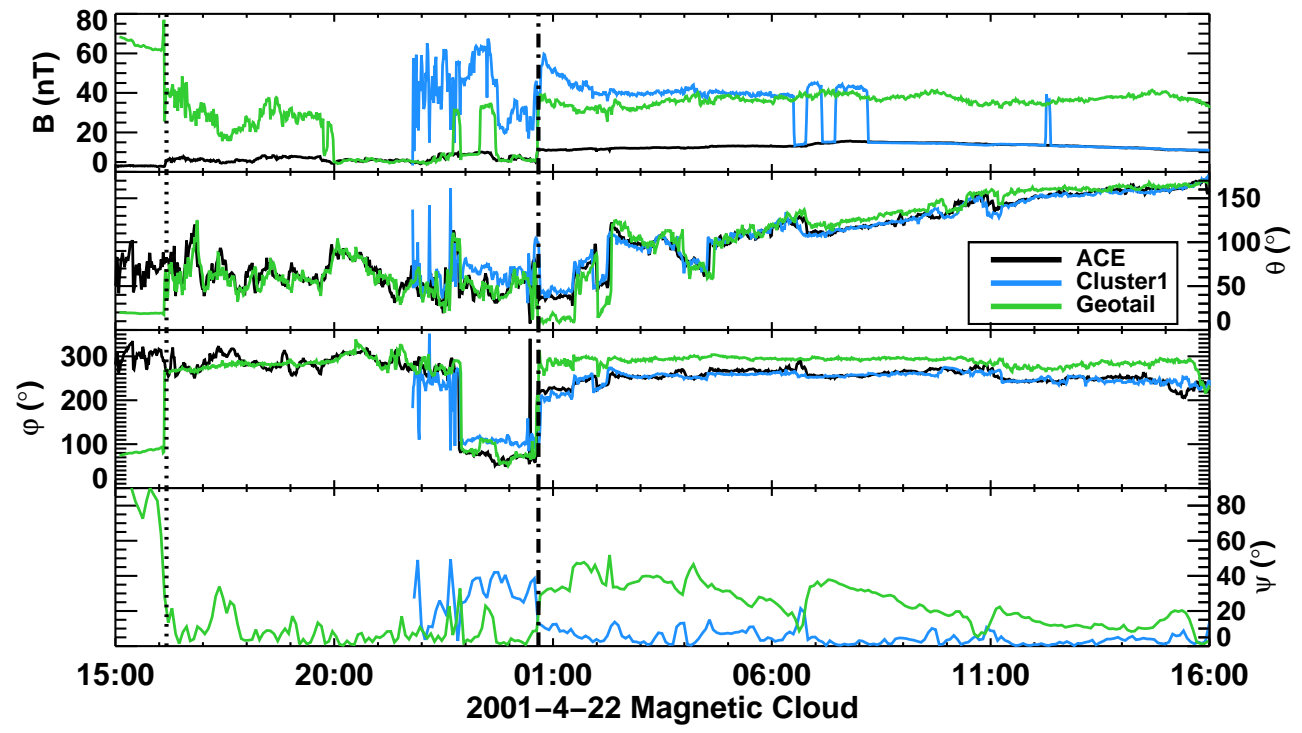

Figure 4. 22 April 2001 MC: observations from ACE (black), Cluster (blue) and Geotail (green), in GSE coordinates. Same format as Fig. 3.

crossing is unclear on the magnetic field data, which display large fluctuations from 16:00 to 22:00 UT (blue curve in Fig. 5a, b and c). Measurements from other instruments aboard the spacecraft, such as the electron and ion fluxes (not shown), indicate that there are in fact several bow shock crossings from 16:30 to 17:15 UT before Cluster remains in the magnetosheath. These crossings are indicated by the vertical red dotted lines in Fig. 5. Around 21:30 UT, Cluster crosses the magnetopause and moves to the magnetosphere. Although this MC event lasts until around 07:00 UT the next day, Fig. 5 is limited to the part of the event relevant for our study, i.e. when Cluster is outside the magnetosphere.
This case differs from the three previous events in several aspects. First, the compression downstream of the bow shock is very weak before 18:30 UT. Although the electron properties show that the spacecraft crosses the bow shock, the magnetic field strength is only slightly enhanced inside the magnetosheath, by a factor of 2 at the most. After 18:30 UT, the magnetic field magnitude increases and fluctuates around $30 \mathrm{nT}$, which is about 3 times its solar wind value. Another distinctive feature of this event is that the MC's smooth rotation is no longer observed downstream of the bow shock (see Fig. 5b and c). The slow variation of $\theta$ and $\varphi$ observed in the solar wind by ACE is blurred by large fluctuations in 


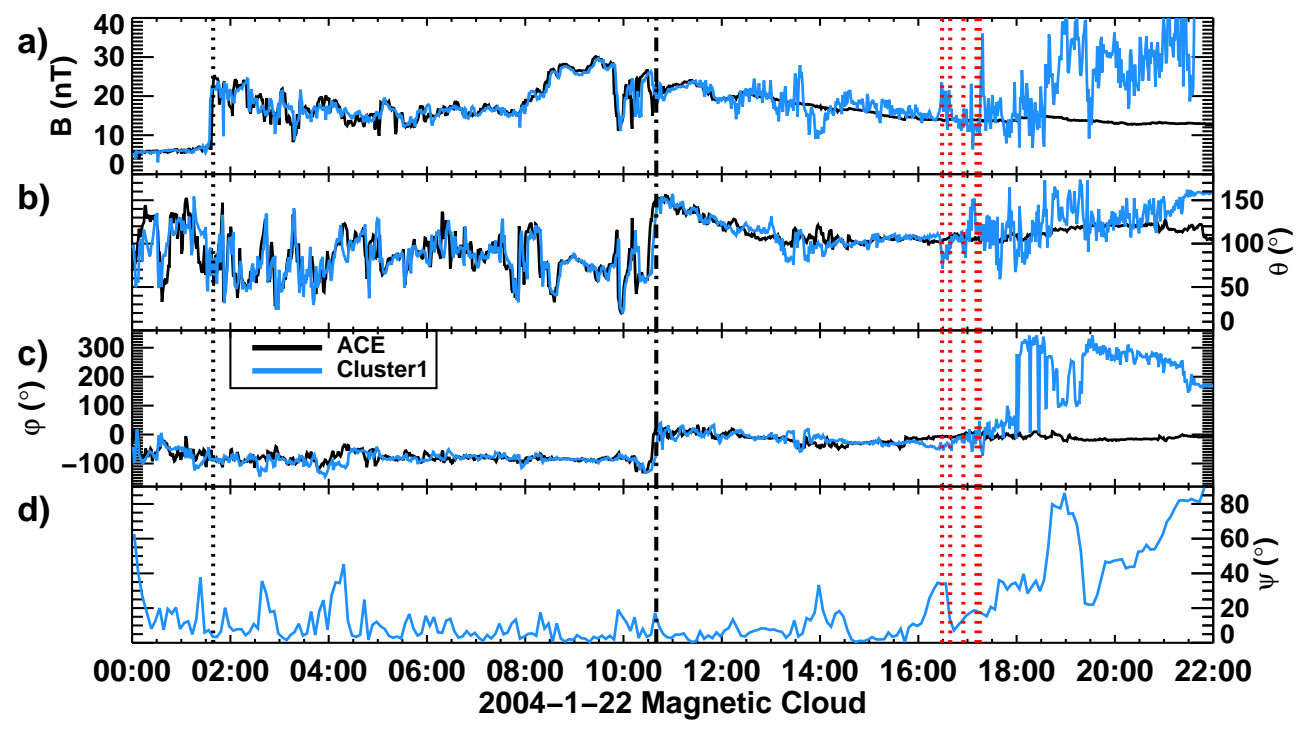

Figure 5. 22 January 2004 MC: observations from ACE (black) and Cluster (blue), in GSE coordinates. Same format as Fig. 3.

magnetic field strength and direction in the magnetosheath. Moreover, we note that these fluctuations are also observed when Cluster is in the solar wind, after 12:00 UT.

$\psi$ (Fig. 5d) illustrates the variation from the solar wind to the magnetosheath of the 5 min averaged magnetic field. $\psi$ is very high during this event, generally above $30^{\circ}$, when Cluster is in the magnetosheath and exceeds $80^{\circ}$ close to the magnetopause and around 19:00 UT. Even on average, the magnetic field direction inside the magnetosheath differs largely from that in the solar wind.

To conclude, we have shown that the evolution of an MC's structure from the solar wind to the magnetosheath differs largely from one event to another. In some cases, the magnetic field direction is preserved across the bow shock (Event 1, second part of Event 2, and Cluster's observations of Event 3 ). In other cases, the smooth rotation closely follows that in the solar wind, but at a different angle (Geotail's observations of Event 3). Finally, the structure of the MC can be strongly modified across the bow shock, as the magnetic field orientation rotates in a somewhat different fashion (first part of Event 2). Large fluctuations in the magnetic field direction can then becloud the smooth rotation of the MC inside the magnetosheath (first part of Event 2 and Event 4). In addition, we have noted that the behaviour of the magnetic field direction across the bow shock can vary with time (Event 2), as the MC passes by, and space, depending on the location inside the magnetosheath (Cluster and Geotail observations on both sides of the magnetosheath, Event 3).

\section{Conditions at the bow shock}

In a simple magnetosheath model, Turc et al. (2014) showed that the MC's structure in the magnetosheath is largely controlled by the conditions encountered at the bow shock. These conditions are quantified by one of the key parameters of the bow shock - the shock obliquity, $\Theta_{\mathrm{Bn}}$. It is defined as the angle between the local normal to the shock's surface and the magnetic field direction in the solar wind. The $\Theta_{\mathrm{Bn}}$ values roughly define two regimes, associated with different physics at the bow shock: if $\Theta_{\mathrm{Bn}}$ ranges between 45 and $90^{\circ}(0$ and $45^{\circ}$ ), the shock is in a quasi-perpendicular (quasi-parallel) configuration. An intermediate regime is sometimes defined, i.e. when $\Theta_{\mathrm{Bn}}$ is around $45^{\circ}$, the so-called oblique shock configuration.

The normal to the shock's surface can be estimated locally when a satellite encounters the bow shock. The four example events were precisely chosen because the spacecraft cross the bow shock multiple times, giving us the opportunity to estimate the shock obliquity. According to Rankine-Hugoniot relations, the magnetic field component along the shock's normal is kept unchanged at the bow shock's crossing. We perform here a minimum variance analysis (MVA) on the magnetic field components measured by the spacecraft to determine the normal direction (see, for example, Sonnerup and Scheible, 1998). The magnetic field direction in the solar wind is given by ACE observations, and this combined with the results of MVA yields the value of $\Theta_{B n}$ at the spacecraft location and at the time of the crossing.

During the events presented in Sect. 3, Cluster encountered the bow shock several times. The values of $\Theta_{\mathrm{Bn}}$ estimated from these crossings are listed in Table 1. Note that we only give here the crossings where MVA yields satisfactory results, i.e. when the time window on which MVA is applied does not affect its results and when the eigenvalues of the variance matrix are well distinct, i.e. when the minimum and intermediate eigenvalues differ by at least a factor of 3.5. The Event 4 is not discussed here because the bow 
Table 1. $\Theta_{\mathrm{Bn}}$ at the bow shock crossings estimated with MVA for the first three events. Note that Event 2 is divided into two parts, corresponding to the quasi-parallel and the quasi-perpendicular configuration at the bow shock.

\begin{tabular}{ccc}
\hline Date & $\begin{array}{c}\text { Time } \\
\text { of crossing }(\mathrm{UT})\end{array}$ & $\begin{array}{c}\Theta_{\mathrm{Bn}} \\
\left.{ }^{\circ}\right)\end{array}$ \\
\hline 20 May 2005 & $07: 52: 41$ & 84 \\
20 May 2005 & $08: 26: 55$ & 81 \\
20 May 2005 & $09: 00: 40$ & 64 \\
20 May 2005 & $09: 05: 55$ & 56 \\
20 May 2005 & $11: 35: 24$ & 71 \\
20 May 2005 & $15: 45: 41$ & 78 \\
20 May 2005 & $16: 07: 00$ & 73 \\
20 May 2005 & $16: 10: 55$ & 62 \\
20 May 2005 & $16: 48: 46$ & 61 \\
\hline 15 May 2005 & $07: 06: 35$ & 32 \\
15 May 2005 & $07: 26: 12$ & 30 \\
15 May 2005 & $07: 50: 40$ & 23 \\
15 May 2005 & $07: 53: 55$ & 20 \\
15 May 2005 & $09: 06: 00$ & 30 \\
15 May 2005 & $09: 12: 25$ & 40 \\
15 May 2005 & $09: 21: 10$ & 24 \\
15 May 2005 & $10: 25: 23$ & 19 \\
15 May 2005 & $11: 00: 40$ & 19 \\
15 May 2005 & $11: 04: 35$ & 44 \\
15 May 2005 & $11: 41: 20$ & 15 \\
15 May 2005 & $11: 46: 40$ & 23 \\
15 May 2005 & $13: 22: 57$ & 30 \\
15 May 2005 & $15: 57: 20$ & 26 \\
\hline 15 May 2005 & $18: 59: 20$ & 64 \\
15 May 2005 & $19: 33: 47$ & 66 \\
15 May 2005 & $19: 44: 30$ & 67 \\
15 May 2005 & $21: 51: 34$ & 81 \\
15 May 2005 & $22: 06: 57$ & 84 \\
15 May 2005 & $22: 11: 43$ & 90 \\
\hline 22 Apr 2001 & $06: 30: 18$ & 89 \\
22 Apr 2001 & $06: 47: 40$ & 86 \\
22 Apr 2001 & $07: 27: 24$ & 86 \\
22 Apr 2001 & $12: 21: 38$ & 87 \\
\hline
\end{tabular}

shock crossings cannot be identified in the magnetic field data, since magnetic field fluctuations are of the order of the background magnetic field.

The $\Theta_{\mathrm{Bn}}$ values show that during Event 1 (Sect. 3.1), the bow shock was quasi-perpendicular at Cluster's location $\left(56^{\circ} \leq \Theta_{\mathrm{Bn}} \leq 84^{\circ}\right)$. Similarly, the $\Theta_{\mathrm{Bn}}$ values indicate that the shock encountered by Cluster during Event 3 (Sect. 3.3) and during the second part of Event 2 (Sect. 3.2) is in a quasi-perpendicular configuration $\left(87^{\circ} \leq \Theta_{\mathrm{Bn}} \leq 89^{\circ}\right.$ and $64^{\circ} \leq \Theta_{\mathrm{Bn}} \leq 90^{\circ}$, respectively). In these three cases, we have seen that the MC's magnetic structure remains roughly unchanged from the solar wind to the magnetosheath. Conversely, during the first part of Event 2, the $\Theta_{\mathrm{Bn}}$ values are much lower $\left(15^{\circ} \leq \Theta_{\mathrm{Bn}} \leq 44^{\circ}\right)$, denoting that Cluster encounters a quasi-parallel bow shock. These low $\Theta_{\mathrm{Bn}}$ values are associated with a large variation of the magnetic field direction across the bow shock, and the structure of the MC differs largely from that in the solar wind. Therefore, the conservation of the MC's structure seems to be related to the crossing of a quasi-perpendicular shock, whereas a quasi-parallel configuration gives rise to a modification of its smooth rotation.

\section{Comparison with a magnetosheath model}

The direct determination of the shock obliquity can only be achieved when the spacecraft encounters the bow shock. Between two consecutive crossings, we cannot know with certainty whether the shock configuration has changed or not. More importantly, if the shock obliquity cannot be determined or if the spacecraft remains in the magnetosheath (for example Geotail during Event 3 presented in Sect. 3.3), we have no information about $\Theta_{\mathrm{Bn}}$. In these cases, the magnetosheath observations can be related to the conditions encountered at the bow shock through modelling.

We use here a model of the magnetosheath magnetic field introduced in Turc et al. (2014) and optimised for MC conditions. Utilising the solar wind parameters as inputs, this model computes the magnetic field inside the magnetosheath. The magnetic field just downstream of the bow shock is calculated with Rankine-Hugoniot equations, and thus depends directly on the compression ratio. It is then propagated along the flowlines calculated by the Soucek and Escoubet (2012) model into the magnetosheath. The boundaries of the magnetosheath are given by the Shue et al. (1998) magnetopause and the Jeřáb et al. (2005) bow shock models. The choice of the Jeřáb et al. (2005) model results from a comparative study of bow shock models during low Alfvén Mach number $\left(M_{\mathrm{A}}<5\right)$ conditions, corresponding to MCs' conditions (Turc et al., 2013). More details about the magnetosheath model can be found in Turc et al. (2014).

We use ACE measurements as the input to the magnetosheath model to compute the magnetic field components at the position of the spacecraft (Cluster or Geotail) in the magnetosheath and compare them to the observations. The model gives us access to the shock conditions that the flow passing by the spacecraft encountered upon entering the magnetosheath. Note that we cannot apply the magnetosheath model to Event 2 because the bow shock model fails to correctly reproduce the bow shock position during this event, as was mentioned in Turc et al. (2013). Consequently, the flow pattern inside the magnetosheath cannot be computed during this MC.

\subsection{Event 1: 20 May 2005 MC}

Figure 6 displays the magnetic field strength (Fig. 6a) and direction (Fig. 6b and c) during the 20 May 2005 MC (Event 


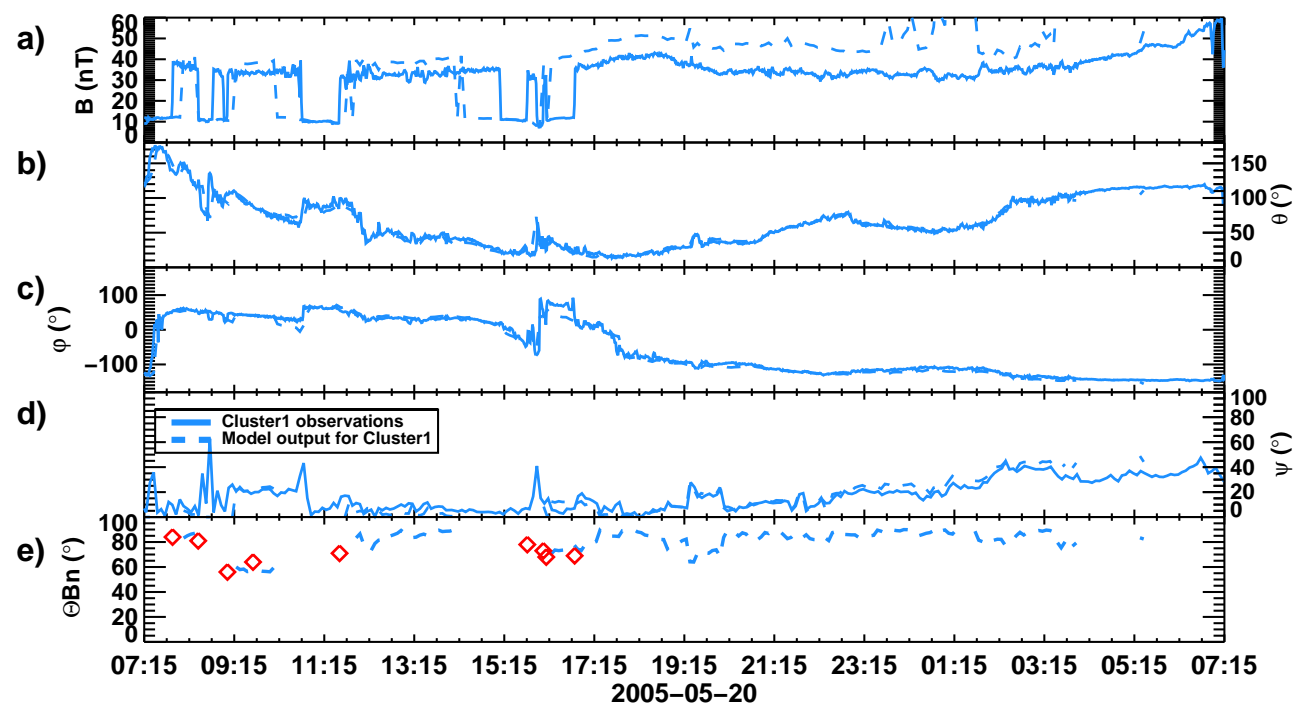

Figure 6. 20 May 2005 MC: comparison between the magnetosheath model's results (dashed blue lines) and Cluster's observations (solid blue lines), in GSE coordinates. From top to bottom: magnetic field strength, $\theta, \varphi, \psi$ and $\Theta_{\mathrm{Bn}}$. Red diamonds: $\Theta_{\mathrm{Bn}}$ estimated with MVA from the observations.

1). It corresponds to the part of Fig. 2 bounded by the two dotted-dashed lines. We show Cluster's observations (solid blue curves) and the magnetosheath model's results (dashed blue curves) during this event. Cluster encounters the shock many times during this event, but the model does not accurately reproduce the times of the bow shock crossings (see Fig. 6a). This is probably due to the bow shock model, based on statistics, which does not take into account the shock's dynamics. However, on average, the bow shock model correctly estimates in which region - magnetosheath or solar wind the spacecraft is located. Around 04:00 UT on 21 May 2005, the magnetopause model predicts that Cluster crosses the magnetopause, which is consistent with the observations.

Inside the magnetosheath, the modelled magnetic field strength is slightly overestimated, in particular after 16:30 UT, but on the whole it is of the same order as that measured by Cluster. After 23:15 UT, the model predicts large peaks of the magnetic field magnitude which are not observed by the spacecraft. These peaks correspond to decreases in the Alfvén Mach number. The model predicts that the bow shock will retreat sunward, and thus the spacecraft appears to be located closer to the magnetopause relative to the distance between the bow shock and the magnetopause, i.e. in a region of larger magnetic compression. We will not discuss any further the discrepancies between the observed and modelled magnetic field strength because they are due to the combined effects of the approximations made in the magnetosheath model such as the compression ratio at the bow shock, the magnitude of the flow velocity, or the density profile inside the magnetosheath (see Turc et al., 2014).

The magnetic field direction given by the model is in excellent agreement with the magnetosheath observations, as shown by the angles $\theta$ and $\varphi$ (Fig. $6 \mathrm{~b}$ and c), as well as the angle $\psi$ between the magnetic field vectors in the solar wind as measured by ACE and in the magnetosheath (Fig. 6d). Figure $6 \mathrm{e}$ shows the $\Theta_{\mathrm{Bn}}$ values encountered at the bow shock at the location connected to the spacecraft along the flowline. Since $\Theta_{\mathrm{Bn}}$ is not defined upstream of the bow shock, the model only provides us with $\Theta_{\mathrm{Bn}}$ values when it predicts the spacecraft to be in the magnetosheath. The results of the model are drawn as a blue dashed curve. They are consistent with the $\Theta_{B n}$ values estimated from Cluster's measurements when the spacecraft crosses the bow shock, indicated by the red diamonds. In particular, it reproduces well the decrease in $\Theta_{\mathrm{Bn}}$ to about $60^{\circ}$ around 09:30 UT and to about $70^{\circ}$ around 16:30 UT. The model results show that the $\Theta_{\mathrm{Bn}}$ remains mostly close to $90^{\circ}$ after Cluster's last bow shock crossing around 16:45 UT.

If we compare $\psi$ and $\Theta_{\mathrm{Bn}}$ (Fig. 6d and e), we find that the variations in $\psi$ seem to be anti-correlated to that of $\Theta_{\mathrm{Bn}}$ during the first half of the event, approximately until 22:00 UT on 20 May 2005. In particular, we note that the deviation between the magnetic field directions observed in the solar wind and in the magnetosheath from 09:00 to 11:00 UT on 20 May 2005, already mentioned in Sect. 3.1, corresponds to a decrease in $\Theta_{\mathrm{Bn}}$. However, the higher $\psi$ values after 22:00 UT cannot be related to a variation of the shock obliquity, since $\Theta_{\mathrm{Bn}}$ remains close to $90^{\circ}$. According to the magnetosheath model, which reproduces these enhanced $\psi$ values well, this increase is due to the field line draping around the magnetosphere. Therefore, the magnetosheath model allows us to separate the impact of the shock obliquity from the effects of the draping on the magnetic field direction inside the magnetosheath. 


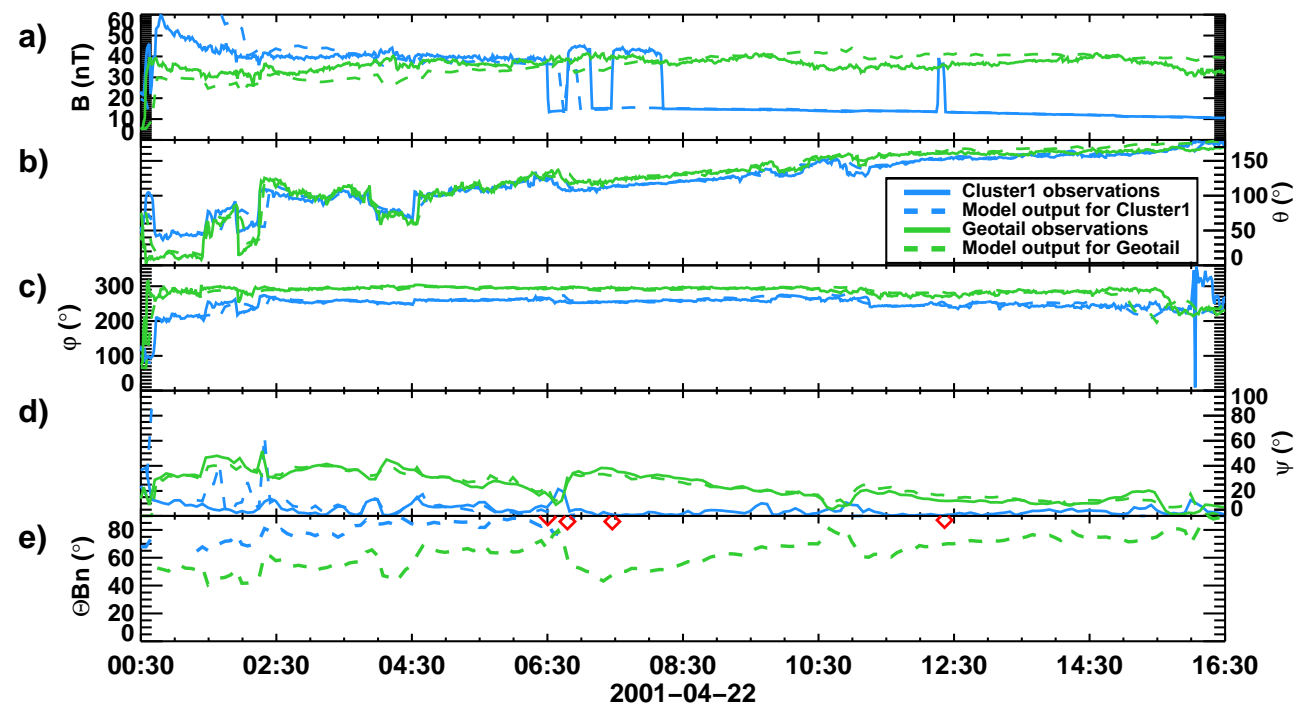

Figure 7. 22 April 2001 MC: comparison between the magnetosheath model's results (dashed lines) and the observations (solid lines) at Cluster's (blue lines) and Geotail's (green lines) locations, in GSE coordinates. Same format as Fig. 6.

\subsection{Event 3: 22 April 2001 MC}

Figure 7 shows the results of the magnetosheath model during the 22 April $2001 \mathrm{MC}$ at Cluster (dashed blue curves) and Geotail (dashed green curves) locations, together with Cluster (solid blue curves) and Geotail (solid green curves) observations. The plotted interval corresponds to the part on the right of the dotted-dashed line in Fig. 4. Cluster is found in the dawnside magnetosheath at the beginning of the $\mathrm{MC}$, and then it moves to the solar wind after 08:30 UT. Geotail remains in the duskside magnetosheath during the entire interval displayed in Fig. 7. Again, the model provides a correct order of magnitude for the magnetic field strength inside the magnetosheath. As in the previous case, the modelled magnetic field direction is in excellent agreement with the observations (see Fig. 7b and c), and the angle $\psi$ (Fig. 7d) shows that the model fits well with the observations for both spacecraft. $\psi$ remains very small at Cluster's location, and decreases from 40 to $20^{\circ}$ at Geotail's.

Figure 7e displays the values of $\Theta_{\mathrm{Bn}}$ upstream of Geotail (dashed green curve) and Cluster (dashed blue curve) when the spacecraft are located downstream of the modelled bow shock. The model shows that Cluster is found downstream of a quasi-perpendicular shock during the first part of the MC $\left(\Theta_{\mathrm{Bn}} \geq 65^{\circ}\right)$ before moving to the solar wind. The comparison between observed and modelled $\Theta_{\mathrm{Bn}}$ is limited, since there are only four $\Theta_{B n}$ values calculated with MVA (red diamonds in panel e) because Cluster crosses the bow shock only a few times, but a very good agreement is obtained. The values of the shock obliquity upstream of Geotail are lower, ranging between 40 and $70^{\circ}$ during the first half of the event. Geotail is then found downstream of a shock in an oblique configuration.
The differences observed between Cluster and Geotail measurements could also be interpreted in terms of their location inside the magnetosheath relative to the magnetopause and the bow shock, since the angle $\psi$ would increase due to field line draping when moving closer to the magnetopause. We computed the angle $\psi$ along the same flowlines as the spacecraft but just downstream of the bow shock, where there is no draping effect. The changes are negligible for Cluster; for Geotail, the angle $\psi$ roughly exhibits the same variations, but with slightly smaller values. However, the contribution of the draping to the encountered $\psi$ values never exceeds $10^{\circ}$. This confirms that the $\psi$ values are higher at Geotail's location than at Cluster's, mainly because the $\Theta_{\mathrm{Bn}}$ values upstream of this spacecraft are lower.

Finally, we note that the variations in $\psi$ and $\Theta_{\mathrm{Bn}}$ are anticorrelated, as was observed in Event 1. This anti-correlation is particularly noticeable on Geotail data because the variations in these two parameters can be compared for 16 consecutive hours as the spacecraft remains continuously in the magnetosheath (green curves in Fig. 7d and e). This suggests that the variation of the magnetic field direction from the solar wind to the magnetosheath is a function of the $\Theta_{\mathrm{Bn}}$ values.

\subsection{Event 4: 22 January 2004 MC}

Cluster's observations (solid blue curves) during the 22 January $2004 \mathrm{MC}$, from 10:30 to 21:30 UT, are displayed in Fig. 8 together with the outputs of the magnetosheath model computed at the spacecraft's location (dashed black curves). Again, this coincides with the part of Fig. 5 on the right of the vertical dotted-dashed line. During this event, Cluster observes very large fluctuations in the magnetic field during the 


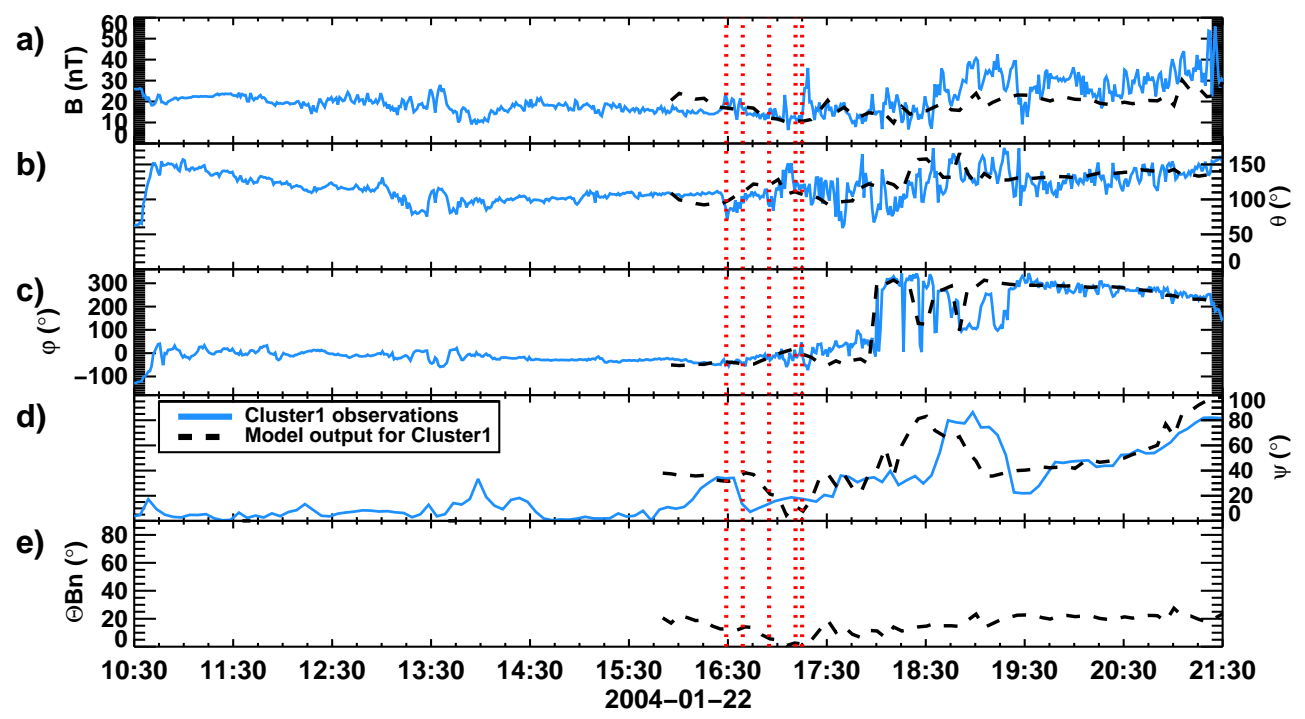

Figure 8. 22 January 2004 MC: comparison between the magnetosheath model's results (dashed black lines) and Cluster's observations (solid blue lines), in GSE coordinates. Same format as Fig. 6.

MC, both upstream and downstream of the bow shock. Because of these fluctuations, the bow shock crossings are unclear in the magnetic field data, but can be identified with the plasma properties. The model predicts the spacecraft to cross the bow shock around 15:45 UT, that is, about $45 \mathrm{~min}$ before the actual crossings, which are marked by the red dotted lines. As mentioned previously, this is due to the limitations of the bow shock model.

First, we note that the large fluctuations in the magnetic field magnitude and direction observed in Cluster data (solid blue curves) are not reproduced by the model (dashed black curves). Therefore, we will mostly discuss the average trend of the magnetic field measurements. On average, the modelled magnetic field strength is of the same order of magnitude as that measured by the spacecraft (Fig. 8a), although the model tends to underestimate it. In terms of the magnetic field direction, the $\theta$ and $\varphi$ obtained with the model are rather close to the average values of the measured $\theta$ and $\varphi$ (Fig. 8b and $\mathrm{c}$ ).

Similarly, the $\psi$ angle (Fig. 8d) given by the model (black curve) reproduces the measurements (blue curve) rather well, most likely because it is calculated from $5 \mathrm{~min}$ averages, which smooth the fluctuations. The major difference between the two curves in Fig. 8d is the position of the peak around 18:30-19:00 UT. According to the magnetosheath model, the increase in $\psi$ up to $80^{\circ}$ around 18:30 UT coincides with an outward motion of the magnetopause and the bow shock caused by a decrease in the Alfvén Mach number (not shown). Because of the displacement of the boundaries, the spacecraft is located much deeper inside the magnetosheath from 18:00 to 19:00 UT. Therefore, the peak of $\psi$ is probably due to the draping of the field lines. What causes the time shift between the modelled (around 18:30 UT) and observed (around 19:00 UT) enhancements of $\psi$ is not clear, however. The decrease in the Alfvén Mach number occurs shortly before 18:00 UT at ACE, and should take less than $1 \mathrm{~h}$ to travel from L1 to the bow shock, according to the solar wind speed. The time lag between the two peaks may stem from the dynamics of the boundaries, which are not properly taken into account in the models, as demonstrated by the bow shock crossing predicted ahead of time. Apart from this peak, the modelled $\psi$ values are quite similar to those provided by Cluster data. In particular, the increase from 40 to $80^{\circ}$ after 19:30 UT obtained with the model is in very good agreement with the observations.

As indicated by the $\Theta_{\mathrm{Bn}}$ values (Fig. 8e), the spacecraft is located downstream of a strongly quasi-parallel shock: $\Theta_{\mathrm{Bn}}$ remains around $20^{\circ}$ from $15: 30$ to $21: 30$ UT. The large variation of the mean magnetic field direction across the bow shock, denoted by the high $\psi$ values, above $30^{\circ}$, is most likely related to these low $\Theta_{\mathrm{Bn}}$ values. At the end of the plot, however, the increase in $\psi$ is not accompanied by variations in $\Theta_{\mathrm{Bn}}$. Therefore, we infer that it is related to the draping of the field lines because at this time Cluster approaches the magnetopause, as was the case in Event 1. Since we are in a quasi-parallel configuration, the large fluctuations in the magnetic field observed by Cluster are expected (see, for example, Shevyrev and Zastenker, 2005; Shevyrev et al., 2007). In particular, from 12:00 to 16:00 UT, when Cluster is in the solar wind, it in fact probes the foreshock, which interacts with the MC. Thus the smoothly rotating magnetic field of the MC can already be altered upstream of the bow shock.

As mentioned in Turc et al. (2014), the magnetosheath model is not expected to reproduce the fluctuations observed downstream of the quasi-parallel shock, or upstream in the foreshock, because it does not take into account the 
microphysical processes taking place there. However, Fig. 8 shows that the average magnetic field direction seems to be reasonably well estimated by the model, even for such low $\Theta_{\mathrm{Bn}}$ values. Regardless, we cannot reasonably use the magnetosheath model to further interpret the observations in this shock configuration.

\section{Discussion and conclusions}

The Earth's bow shock is known to modify the properties of the incoming solar wind to divert it around the magnetosphere. In particular, the interplanetary magnetic field (IMF) magnitude increases and its direction rotates as predicted by the Rankine-Hugoniot relations and due to the field line draping around the magnetopause. The same processes are expected to occur when an MC crosses the bow shock. This could significantly modify the orientation of the MC's magnetic field and consequently alter its interaction with the magnetosphere through reconnection or other local processes. However, the effects of the bow shock on the MC's magnetic structure are generally not taken into account when studying the geoeffectivity of MCs.

In this paper, we analyse spacecraft observations in the Earth's magnetosheath to investigate the variation of the magnetic structure of MCs caused by the bow shock's crossing. We study four events as four typical and different examples of magnetosheath observations during MCs. In the first case, the MC's structure observed in the magnetosheath is roughly the same as that in the solar wind. In contrast, the second event highlights that the MC's smooth rotation sometimes differs largely from that observed upstream of the bow shock. Moreover, the alteration of the MC's structure across the bow shock can vary with time as the MC passes by the Earth. In the third example, data from two different spacecraft are available simultaneously at two different locations inside the magnetosheath. The combined observations of Cluster and Geotail show that the structure of the MC differs from one region of the magnetosheath to another during this event. In the dawnside magnetosheath, the magnetic field orientation is the same as in the solar wind, whereas in the duskside it follows the smooth rotation observed upstream, but at a different angle. Finally, the fourth event illustrates that, in some cases, the MC's smooth rotation disappears inside the magnetosheath because of large magnetic field fluctuations. In this case, the magnetic compression downstream of the bow shock is also very weak.

The differences between these four events appear to be strongly related to the conditions encountered at the bow shock. The values of the shock obliquity, $\Theta_{\mathrm{Bn}}$, are determined directly with minimum variance analysis when bow shock crossings are observed, or indirectly with a magnetosheath model (Turc et al., 2014). A good agreement between the model's results and the observations is obtained. We find that, when $\Theta_{\mathrm{Bn}}$ is close to $90^{\circ}$, the structure of the $\mathrm{MC}$ is roughly conserved downstream of the bow shock. As $\Theta_{\mathrm{Bn}}$ decreases to more oblique values, the variation of the magnetic field direction from the solar wind to the magnetosheath increases. If the shock is in a quasi-parallel configuration, the MC's structure inside the magnetosheath differs significantly from that in the solar wind. In Event 2, the modification of the magnetic field orientation can exceed $60^{\circ}$ just downstream of the bow shock. The temporal variation of the difference between upstream and downstream structures, observed during the 15 May 2005 MC (Event 2), can be related to the timevarying shock configuration, which progressively turns from quasi-parallel to quasi-perpendicular at Cluster's location as the MC moves past the Earth.

The results of this case study stress the important role played by the shock configuration on the MC's structure inside the magnetosheath. This is consistent with the conclusions drawn by Turc et al. (2014) when applying the magnetosheath model to synthetic MCs. On the basis of the observations of the magnetic structure of MCs in the magnetosheath, we suggest that three different scenarios can occur at the bow shock's crossing, depending on the encountered shock configuration, i.e. quasi-perpendicular, oblique or quasi-parallel. If the shock is in a quasi-perpendicular configuration (i.e. $\Theta_{\mathrm{Bn}} \gtrsim 60^{\circ}$ ), the MC's structure is almost unchanged at the bow shock's crossing, that is, $\psi$ is below $20^{\circ}$. In an oblique configuration (i.e. $60^{\circ} \gtrsim \Theta_{\mathrm{Bn}} \gtrsim 30^{\circ}$ ), the magnetic field orientation varies significantly $\left(\psi>20^{\circ}\right)$ but still follows a similar smooth rotation as in the solar wind. Finally, if the MC encounters a quasi-parallel shock (i.e. $\Theta_{\mathrm{Bn}} \lesssim 30^{\circ}$ ), the magnetic field orientation again varies largely, but in addition its slow variation changes from the solar wind to the magnetosheath, where fluctuations arise and dominate any structured magnetic field. As the quasi-parallel and the quasi-perpendicular regimes can coexist on the bow shock's surface, it is likely that the MC's structure will be strongly modified in a part of the magnetosheath. The magnetic field reaching the magnetopause during the MC's passage will most likely display pronounced asymmetries, in the same way as the Parker-spiral IMF creates dawn-dusk asymmetries, which could have an impact on its interaction and possible reconnection with the Earth's magnetic field. To investigate this in more detail, it could be worth considering an extension of the Turc et al. (2014) magnetosheath model which includes reconnection processes, as is done for example in Cooling et al. (2001), but this lies beyond the scope of this paper.

In addition to the interpretation of the observations of MCs inside the magnetosheath, the comparison of the model's results with the spacecraft measurements allows for the interests and limitations of the model to be discussed. Firstly, the magnetic field strength obtained with the model is of the same order of magnitude as that observed by the satellites. It is sometimes slightly overestimated or underestimated, and it strongly depends on the approximations made in the flow model and in the compression ratio applied at the bow shock. 
Finding the precise source of these discrepancies is left for future work. Secondly, the modelled magnetic field direction is in excellent agreement with the observations. The same conclusions are drawn for the values of $\Theta_{\mathrm{Bn}}$ when the spacecraft cross the bow shock. We interpret the variations in the magnetic field direction from the solar wind to the magnetosheath as a function of the conditions encountered at the bow shock, that is, the $\Theta_{\mathrm{Bn}}$ values. In some cases, when $\Theta_{\mathrm{Bn}}$ cannot be invoked to account for the observed variations, the model allows us to relate them to the field line draping. We also find that the magnetosheath model yields very good results even at two distant locations inside the magnetosheath, and allows us to consistently interpret the differences between the two spacecraft observations. Thirdly, we note that the bow shock model does not reproduce the timing of the bow shock crossings exactly. This is most likely due to the fact that the bow shock model is built on statistical data sets of the bow shock position, but does not include its dynamics. Moreover, MCs correspond to rather extreme solar wind conditions, during which the accuracy of the model is expected to decrease. Therefore, there is a need for a more accurate parametrisation of the bow shock position and dynamics during MC conditions, but again this lies beyond the scope of this paper. Finally, the magnetosheath model is not reliable when the $\Theta_{\mathrm{Bn}}$ values are particularly low because it does not reproduce the magnetic field fluctuations and the turbulence associated with the quasi-parallel shock. However we find a rather good agreement between the average modelled and observed magnetic fields.

Therefore, even if no direct estimate of the bow shock normal is available, we can rely on this magnetosheath model to determine the shock obliquity upstream of the satellite, as was done in Sect. 5. This provides us with the means to relate dayside magnetosheath observations during $\mathrm{MC}$ events to the conditions encountered at the shock. Additionally, this model allows for the impact of the bow shock to be separated from the effects of the draping on the magnetic field direction.

Finally, the present study evidences that, in some cases, the MC's magnetic field varies significantly from the solar wind to the magnetosheath. Therefore, during such events, the magnetic field impinging on the magnetopause cannot be approximated by the IMF, and the magnetosheath magnetic field should be taken into account. This large variation of the magnetic field orientation occurs when an oblique or quasi-parallel configuration is encountered at the bow shock's crossing. For that reason, the knowledge of the shock obliquity seems to be crucial to better understand the impact of an MC on the Earth's environment.

Acknowledgements. The authors thank the ACE SWEPAM and MAG, the GEOTAIL MGF, and the Cluster FGM and PEACE instrument teams. We also thank the Coordinated Data Analysis Web (CDAWeb) service for providing ACE and GEOTAIL data, and the Cluster Active Archive for providing Cluster data. E. Kilpua acknowledges Academy of Finland projects 1267087 and 1218152.
Topical Editor C. Owen thanks two anonymous referees for their help in evaluating this paper.

\section{References}

Acuña, M. H., Ogilvie, K. W., Baker, D. N., Curtis, S. A., Fairfield, D. H., and Mish, W. H.: The Global Geospace Science Program and Its Investigations, Space Sci. Rev., 71, 5-21, doi:10.1007/BF00751323, 1995.

Angelopoulos, V.: The THEMIS Mission, Space Sci. Rev., 141, 534, doi:10.1007/s11214-008-9336-1, 2008

Balogh, A., Dunlop, M. W., Cowley, S. W. H., Southwood, D. J., Thomlinson, J. G., Glassmeier, K. H., Musmann, G., Luhr, H., Buchert, S., Acuna, M. H., Fairfield, D. H., Slavin, J. A., Riedler, W., Schwingenschuh, K., and Kivelson, M. G.: The Cluster Magnetic Field Investigation, Space Sci. Rev., 79, 6591, doi:10.1023/A:1004970907748, 1997.

Bisi, M. M., Breen, A. R., Jackson, B. V., Fallows, R. A., Walsh, A. P., Mikić, Z., Riley, P., Owen, C. J., Gonzalez-Esparza, A., Aguilar-Rodriguez, E., Morgan, H., Jensen, E. A., Wood, A. G., Owens, M. J., Tokumaru, M., Manoharan, P. K., Chashei, I. V., Giunta, A. S., Linker, J. A., Shishov, V. I., Tyul'Bashev, S. A., Agalya, G., Glubokova, S. K., Hamilton, M. S., Fujiki, K., Hick, P. P., Clover, J. M., and Pintér, B.: From the Sun to the Earth: The 13 May 2005 Coronal Mass Ejection, Sol. Phys., 265, 49127, doi:10.1007/s11207-010-9602-8, 2010

Burlaga, L., Sittler, E., Mariani, F., and Schwenn, R.: Magnetic loop behind an interplanetary shock - Voyager, Helios, and IMP 8 observations, J. Geophys. Res., 86, 6673-6684, doi:10.1029/JA086iA08p06673, 1981

Cooling, B. M. A., Owen, C. J., and Schwartz, S. J.: Role of the magnetosheath flow in determining the motion of open flux tubes, J. Geophys. Res., 106, 18763-18775, doi:10.1029/2000JA000455, 2001

Dasso, S., Mandrini, C. H., Schmieder, B., Cremades, H., Cid, C., Cerrato, Y., Saiz, E., Démoulin, P., Zhukov, A. N., Rodriguez, L., Aran, A., Menvielle, M. and Poedts, S.: Linking two consecutive nonmerging magnetic clouds with their solar sources, J. Geophys. Res., 114, A02109, doi:10.1029/2008JA013102, 2009

Echer, E., Gonzalez, W. D., Tsurutani, B. T. and Gonzalez, A. L. C.: Interplanetary conditions causing intense geomagnetic storms (Dst $\leq-100 \mathrm{nT}$ ) during solar cycle 23 (1996-2006), J. Geophys. Res., 113, A05221, doi:10.1029/2007JA012744, 2008

Escoubet, C. P., Schmidt, R., and Goldstein, M. L.: Cluster Science and Mission Overview, Space Sci. Rev., 79, 11-32, doi:10.1023/A:1004923124586, 1997.

Gopalswamy, N., Akiyama, S., Yashiro, S., Michalek, G. and Lepping, R. P.: Solar sources and geospace consequences of interplanetary magnetic clouds observed during solar cycle 23, J. Atmos. Sol.-Terr. Phys., 70, 245-253, doi:10.1016/j.jastp.2007.08.070, 2008.

Huttunen, K. E. J., Schwenn, R., Bothmer, V., and Koskinen, H. E. J.: Properties and geoeffectiveness of magnetic clouds in the rising, maximum and early declining phases of solar cycle 23, Ann. Geophys., 23, 625-641, doi:10.5194/angeo-23-625-2005, 2005.

Jeřáb, M., Němeček, Z., Šafránková, J., Jelínek, K., and Měrka, J.: Improved bow shock model with depen- 
dence on the IMF strength, Planet. Space Sci., 53, 85-93, doi:10.1016/j.pss.2004.09.032, 2005.

Kokubun, S., Yamamoto, T., Acuña, M. H., Hayashi, K., Shiokawa, K., and Kawano, H.: The GEOTAIL Magnetic Field Experiment, J. Geomag. Geoelectr., 46, 7-21, 1994.

Lavraud, B. and Borovsky, J. E.: Altered solar wind-magnetosphere interaction at low Mach numbers: Coronal mass ejections, J. Geophys. Res., 113, A00B08, doi:10.1029/2008JA013192, 2008.

Lavraud, B., Larroque, E., Budnik, E., Génot, V., Borovsky, J. E., Dunlop, M. W., Foullon, C., Hasegawa, H., Jacquey, C., Nykyri, K., Ruffenach, A., Taylor, M. G. G. T., Dandouras, I., and Rème, H.: Asymmetry of magnetosheath flows and magnetopause shape during low Alfvén Mach number solar wind, J. Geophys. Res., 118, 1089-1100, doi:10.1002/jgra.50145, 2013.

Liu, Z. X., Escoubet, C. P., Pu, Z., Laakso, H., Shi, J. K., Shen, C. and Hapgood, M.: The Double Star mission, Ann. Geophys., 23, 2707-2712, doi:10.5194/angeo-23-2707-2005, 2005.

Longmore, M., Schwartz, S. J., and Lucek, E. A.: Rotation of the magnetic field in Earth's magnetosheath by bulk magnetosheath plasma flow, Ann. Geophys., 24, 339-354, doi:10.5194/angeo24-339-2006, 2006.

Nishida, A.: The GEOTAIL mission, Geophys. Res. Lett., 21, 28712873, doi:10.1029/94GL01223, 1994.

Richardson, I. G. and Cane, H. V.: Near-Earth Interplanetary Coronal Mass Ejections During Solar Cycle 23 (1996 - 2009): Catalog and Summary of Properties, Solar Physics, 264, 189-237, doi:10.1007/s11207-010-9568-6, 2010.

Richardson, I. G., Cliver, E. W., and Cane, H. V.: Sources of geomagnetic storms for solar minimum and maximum conditions during 1972-2000, Geophys. Res. Lett., 28, 2569-2572, doi:10.1029/2001GL013052, 2001.

Richardson, I. G., Cane, H. V., and Cliver, E. W.: Sources of geomagnetic activity during nearly three solar cycles (1972-2000), J. Geophys. Res., 107, 1187, doi:10.1029/2001JA000504, 2002.

Šafránková, J., Hayosh, M., Gutynska, O., Němeček, Z., and Přech, L.: Reliability of prediction of the magnetosheath $\mathrm{B}_{Z}$ component from interplanetary magnetic field observations, J. Geophys. Res., 114, A12213, doi:10.1029/2009JA014552, 2009.

Shevyrev, N. N. and Zastenker, G. N.: Some features of the plasma flow in the magnetosheath behind quasi-parallel and quasi-perpendicular bow shocks, Planet. Space Sci., 53, 95-102, doi:10.1016/j.pss.2004.09.033, 2005.
Shevyrev, N. N., Zastenker, G. N., and Du, J.: Statistics of lowfrequency variations in solar wind, foreshock and magnetosheath: INTERBALL-1 and CLUSTER data, Planet. Space Sci., 55, 2330-2335, doi:10.1016/j.pss.2007.05.014, 2007.

Shue, J.-H., Song, P., Russell, C. T., Steinberg, J. T., Chao, J. K., Zastenker, G., Vaisberg, O. L., Kokubun, S., Singer, H. J., Detman, T. R., and Kawano, H.: Magnetopause location under extreme solar wind conditions, J. Geophys. Res., 103, 17691-17700, doi:10.1029/98JA01103, 1998.

Smith, C. W., L'Heureux, J., Ness, N. F., Acuña, M. H., Burlaga, L. F., and Scheifele, J.: The ACE Magnetic Fields Experiment, Space Sci. Rev., 86, 613-632, doi:10.1023/A:1005092216668, 1998.

Sonnerup, U. Ö. and Scheible, M.: Minimum and maximum variance analysis, in: Analysis Methods for Multi-spacecraft Data, edited by: Paschmann, G. and Daly, W., ISSI Scientific Report, 1998.

Soucek, J. and Escoubet, C. P.: Predictive model of magnetosheath plasma flow and its validation against Cluster and THEMIS data, Ann. Geophys., 30, 973-982, doi:10.5194/angeo-30-973-2012, 2012.

Spreiter, J. R., Summers, A. L., and Alksne, A. Y.: Hydromagnetic flow around the magnetosphere, Planet. Space Sci., 14, 223-253, doi:10.1016/0032-0633(66)90124-3, 1966.

Stone, E. C., Frandsen, A. M., Mewaldt, R. A., Christian, E. R., Margolies, D., Ormes, J. F., and Snow, F.: The Advanced Composition Explorer, Space Sci. Rev., 86, 1-22, doi:10.1023/A:1005082526237, 1998.

Turc, L., Fontaine, D., Savoini, P., Hietala, H., and Kilpua, E. K. J.: A comparison of bow shock models with Cluster observations during low Alfvén Mach number magnetic clouds, Ann. Geophys., 31, 1011-1019, doi:10.5194/angeo-31-1011-2013, 2013.

Turc, L., Fontaine, D., Savoini, P., and Kilpua, E. K. J.: A model of the magnetosheath magnetic field during magnetic clouds, Ann. Geophys., 32, 157-173, doi:10.5194/angeo-32-157-2014, 2014.

Yermolaev, Y. I., Nikolaeva, N. S., Lodkina, I. G., and Yermolaev, M. Y.: Geoeffectiveness and efficiency of CIR, sheath, and ICME in generation of magnetic storms, J. Geophys. Res., 117, A00L07, doi:10.1029/2011JA017139, 2012.

Zhang, J., Liemohn, M. W., Kozyra, J. U., Lynch, B. J., and Zurbuchen, T. H.: A statistical study of the geoeffectiveness of magnetic clouds during high solar activity years, J. Geophys. Res., 109, A09101, doi:10.1029/2004JA010410, 2004. 